PROCEEDINGS OF THE

AMERICAN MATHEMATICAL SOCIETY

Volume 129, Number 8, Pages 2301-2308

S 0002-9939(01)05968-8

Article electronically published on February 2, 2001

\title{
THE FUCIK SPECTRUM AND CRITICAL GROUPS
}

\author{
KANISHKA PERERA AND MARTIN SCHECHTER
}

(Communicated by David S. Tartakoff)

\begin{abstract}
We compute critical groups of zero for variational functionals arising from semilinear elliptic boundary value problems with jumping nonlinearities when the asymptotic limits of the nonlinearity fall in certain parts of Type (II) regions between curves of the Fucik spectrum.
\end{abstract}

\section{INTRODUCTION}

In this paper we consider the problem of determining the critical groups of zero for the functional

$$
I(u)=I(u, a, b)=\int_{\Omega}|\nabla u|^{2}-a\left(u^{-}\right)^{2}-b\left(u^{+}\right)^{2}, \quad u \in H=H_{0}^{1}(\Omega),
$$

associated with the problem

$$
\left\{\begin{aligned}
-\Delta u & =b u^{+}-a u^{-} & & \text {in } \Omega, \\
u & =0 & & \text { on } \partial \Omega
\end{aligned}\right.
$$

where $\Omega$ is a smooth bounded domain in $\mathbb{R}^{n}$ and $u^{ \pm}(x)=\max \{ \pm u(x), 0\}$.

The set $\Sigma$ of those points $(a, b) \in \mathbb{R}^{2}$ for which (1.2) has nontrivial solutions is called the Fucik spectrum of $-\Delta$. It was shown in Schechter [7] that, if $0<$ $\lambda_{1}<\lambda_{2}<\cdots$ are the distinct Dirichlet eigenvalues of $-\Delta$, there are decreasing curves $C_{l 1}, C_{l 2}$ (which may coincide) passing through the point $\left(\lambda_{l}, \lambda_{l}\right)$ such that all points on the curves are in $\Sigma$, while points in the square $Q_{l}:=\left(\lambda_{l-1}, \lambda_{l+1}\right)^{2}$ that are either in the region $\mathrm{I}_{l 1}$ below the lower curve $C_{l 1}$ or in the region $\mathrm{I}_{l 2}$ above the upper curve $C_{l 2}$ are not in $\Sigma$. When the curves do not coincide, points in the region $\mathrm{II}_{l}$ between them may or may not belong to $\Sigma$. We set $\mathrm{I}_{l}=\mathrm{I}_{l 1} \cup \mathrm{I}_{l-1,2}$.

When $(a, b) \notin \Sigma$, the origin is an isolated critical point of $I$ and hence the critical groups $C_{*}(I, 0)$ are defined. The following partial result on the critical groups was obtained by Dancer [2, 1].

Theorem 1.1. Let $(a, b) \in Q_{l} \backslash \Sigma$ and let $d_{l}$ denote the dimension of the subspace $N_{l}$ spanned by the eigenfunctions corresponding to $\lambda_{1}, \cdots, \lambda_{l}$.

(i) If $(a, b) \in \mathrm{I}_{l}$, then $C_{q}(I, 0)= \begin{cases}\mathbb{Z}, & q=d_{l-1}, \\ 0, & q \neq d_{l-1} .\end{cases}$

Received by the editors November 26, 1999 .

2000 Mathematics Subject Classification. Primary 35J65, 58E05, 49B27.

(C)2001 American Mathematical Society 
(ii) If $(a, b) \in \mathrm{II}_{l}$, then $C_{q}(I, 0)=0$ for $q \leq d_{l-1}$ and for $q \geq d_{l}$. In particular, $C_{q}(I, 0)=0$ for all $q$ when $\lambda_{l}$ is a simple eigenvalue.

A different proof of Theorem 1.1 based on some ideas developed in Schechter 7.8 was given in Perera and Schechter [5]. Note that this theorem does not determine $C_{q}(I, 0)$ for $d_{l-1}<q<d_{l}$ when $(a, b) \in \mathrm{II}_{l}$ (and $\lambda_{l}$ is a multiple eigenvalue). Here we compute these critical groups for some of the points in $\mathrm{II}_{l}$.

Definition 1.2. For $p \in Q_{l} \cap C_{l 2}$, denote by $\Gamma_{p}$ the set of those points $(a, b) \in \mathbb{R}^{2} \backslash \Sigma$ for which there is a curve $\gamma=\left(\gamma_{1}, \gamma_{2}\right) \in C([0,2]) \cap C^{1}([0,1]), \gamma(0)=p, \gamma(2)=(a, b)$ such that

(i) $\gamma((0,2]) \cap \Sigma=\emptyset$,

(ii) $\gamma_{1}^{\prime}, \gamma_{2}^{\prime} \leq 0$ on $[0,1]$,

(iii) $\gamma(0)+\gamma^{\prime}(0)=\gamma(1)$.

We can take $\gamma$ to be the line segment joining $p=\left(p_{1}, p_{2}\right)$ and $(a, b)$ if it intersects $\Sigma$ only at $p$ and $a \leq p_{1}, b \leq p_{2}$. For example, suppose $C_{l 1}$ and $C_{l 2}$ intersect only at $\left(\lambda_{l}, \lambda_{l}\right)$ and $\mathrm{II}_{l} \cap \Sigma=\emptyset$, as in the figure below. Then the region $\mathrm{II}_{l}$ consists of two connected components. The component to the left (resp. right) of $\left(\lambda_{l}, \lambda_{l}\right)$ is contained in $\Gamma_{p}$ for each $p \in Q_{l} \cap C_{l 2}$ to the left (resp. right) of $\left(\lambda_{l}, \lambda_{l}\right)$. Also note that $\Gamma_{\left(\lambda_{l}, \lambda_{l}\right)}$ always contains $\mathrm{I}_{l}$.

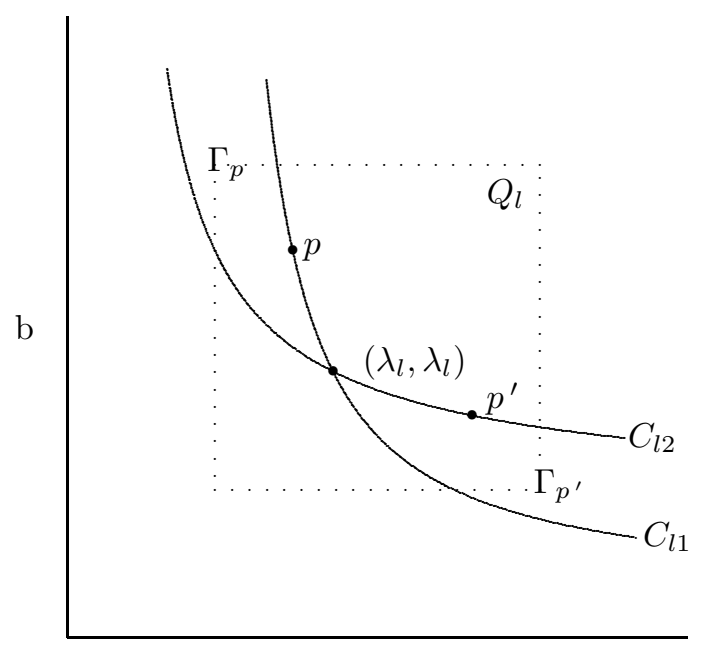

a

Observe that, by (ii) and the homotopy invariance of the critical groups, the $C_{*}(I(\cdot, \gamma(t)), 0)$ are defined for all $t \in(0,2]$ and are independent of $t$. Let $K_{p}$ denote the set of critical points of $I_{p}=I(\cdot, p)$ and let $\widehat{K}_{p}=\left\{u \in K_{p}:\|u\|=1\right\}$.

Theorem 1.3. If $p \in Q_{l} \cap C_{l 2}$ and $(a, b) \in \Gamma_{p}$, then

$$
C_{q}(I, 0) \cong \begin{cases}H^{d_{l}-q-1}\left(\widehat{K}_{p}\right), & q \neq d_{l}-1, \\ H^{0}\left(\widehat{K}_{p}\right) / \mathbb{Z}, & q=d_{l}-1 .\end{cases}
$$

Theorem 1.3 is proved in Section 2 and should be compared with Theorem 2 of Dancer [2]. Note that Theorem [1.3] completely determines the critical groups for all $(a, b) \in \mathrm{II}_{l}$ when the region $\mathrm{II}_{l}$ is free of $\Sigma$. 
As an application we consider the problem

$$
\left\{\begin{aligned}
-\Delta u & =f(x, u) & & \text { in } \Omega, \\
u & =0 & & \text { on } \partial \Omega
\end{aligned}\right.
$$

where $f \in C(\bar{\Omega} \times \mathbb{R})$ and

$$
f(x, t)= \begin{cases}b_{0} t^{+}-a_{0} t^{-}+o(t) & \text { as } t \rightarrow 0 \\ b t^{+}-a t^{-}+o(t) & \text { as } t \rightarrow \infty\end{cases}
$$

with $\left(a_{0}, b_{0}\right),(a, b) \notin \Sigma$. It was shown in Perera and Schechter 4 that this problem has a nontrivial solution if, for some $q$,

$$
C_{q}\left(I_{0}, 0\right) \neq C_{q}(I, 0)
$$

where $I_{0}=I\left(\cdot, a_{0}, b_{0}\right)$. By Theorem 1.1, this is the case when $\left(a_{0}, b_{0}\right) \in \mathrm{I}_{j}$ and $(a, b) \in \mathrm{I}_{l}$ with $j \neq l$ and when $\left(a_{0}, b_{0}\right) \in \mathrm{I}_{j}$ and $(a, b) \in \mathrm{II}_{l}$ (or $(a, b) \in \mathrm{I}_{l}$ and $\left.\left(a_{0}, b_{0}\right) \in \mathrm{II}_{j}\right)$. We use Theorem 1.3 to consider the case where $\left(a_{0}, b_{0}\right) \in \mathrm{II}_{j}$, $(a, b) \in \mathrm{II}_{l}$, and $\lambda_{l}$ is a multiple eigenvalue. Let $i_{q}(p)$ denote the right hand side of (1.3) and let $Q=\bigcup_{l} Q_{l}$.

Theorem 1.4. If $\left(a_{0}, b_{0}\right),(a, b) \notin \Sigma$, then (1.4) has a nontrivial solution in each of the following cases:

(i) $\left(a_{0}, b_{0}\right) \in \Gamma_{p}$ for some $p \in\left(Q_{l} \cap C_{l 2}\right) \backslash C_{l 1}$ with $i_{q}(p) \neq 0$ for some $q$, and $(a, b) \in Q \backslash \mathrm{II}_{l}$,

(ii) $(a, b) \in \Gamma_{p}$ for some $p \in\left(Q_{l} \cap C_{l 2}\right) \backslash C_{l 1}$ with $i_{q}(p) \neq 0$ for some $q$, and $\left(a_{0}, b_{0}\right) \in Q \backslash \mathrm{II}_{l}$.

Note that, since $p \notin C_{l 1}$ and $\gamma_{1}^{\prime}, \gamma_{2}^{\prime} \leq 0$ on $[0,1], \gamma(t) \in \mathrm{II}_{l}$ for $t>0$ sufficiently small, and hence $i_{q}(p) \neq 0$ implies $d_{l-1}<q<d_{l}$ by the remark following Definition 1.2 and part (ii) of Theorem 1.1. For example, $i_{d_{l}-1}(p) \neq 0$ if $\widehat{K}_{p}$ is disconnected (this may be caused by a symmetry of $\Omega$, and hence of $I_{p}$ ).

Theorem 1.3 also implies that $H^{*}\left(\widehat{K}_{p_{1}}\right) \cong H^{*}\left(\widehat{K}_{p_{2}}\right)$ whenever $p_{1}, p_{2} \in Q_{l} \cap C_{l 2}$ and $\Gamma_{p_{1}} \cap \Gamma_{p_{2}} \neq \emptyset$. Moreover, it can be combined with Theorem 1.1 to obtain extra information on $K_{p}$. Let $M_{l}=N_{l}^{\perp}$. It was shown in Schechter $[8]$ that there are continuous and positive homogeneous functions $\tau: N_{l} \rightarrow M_{l}, \theta: M_{l-1} \rightarrow N_{l-1}$ such that $w_{0}=\tau(v), v_{0}=\theta(w)$ are the unique solutions of

$$
\begin{gathered}
I_{p}\left(v+w_{0}\right)=\inf _{w \in M_{l}} I_{p}(v+w), \quad v \in N_{l}, \\
I_{p}\left(v_{0}+w\right)=\sup _{v \in N_{l-1}} I_{p}(v+w), \quad w \in M_{l-1},
\end{gathered}
$$

respectively. Let

$$
\begin{gathered}
S_{l 1}=\left\{v+\tau(v): v \in N_{l}\right\}, \\
S_{l 2}=\left\{\theta(w)+w: w \in M_{l-1}\right\}, \\
S_{l}=S_{l 1} \cap S_{l 2} .
\end{gathered}
$$

We will see in Section 3 that $S_{l}$ is a radial manifold modeled on the eigenspace $E\left(\lambda_{l}\right)$. Let $\widehat{S}_{l}=\widehat{S}_{l}(p)=\left\{u \in S_{l}:\|u\|=1\right\}$. It follows from Lemmas 3.10 and 3.16 of Schechter [7] that $\widehat{K}_{p} \subset \widehat{S}_{l}$. Moreover, by Lemmas 3.6 and 3.7 of Schechter [6], $I_{p} \leq 0$ on $S_{l 1}$ and $K_{p}=S_{l 1} \cap I_{p}^{-1}(0)$ if $p \in C_{l 2}$, while $I_{p} \geq 0$ on $S_{l 2}$ and $K_{p}=S_{l 2} \cap I_{p}^{-1}(0)$ if $p \in C_{l 1}$, so $\widehat{K}_{p}=\widehat{S}_{l}$ if $p \in Q_{l} \cap C_{l 1} \cap C_{l 2}$. We shall prove 
Theorem 1.5. Let $p \in\left(Q_{l} \cap C_{l 2}\right) \backslash C_{l 1}$. If $\Gamma_{p} \neq \emptyset$, then $\widehat{K}_{p}$ is a proper subset of $\widehat{S}_{l}$. In particular, $\widehat{K}_{p}$ is a single point if $\lambda_{l}$ is simple.

As we will see in Section 2 the proof of Theorem 1.3 involves considering $\left.I\right|_{S_{l 1}}$. We suspect that there is a counterpart of this theorem for the lower curve $C_{l 1}$, but the proof seems to be more complicated because the manifold $S_{l 2}$ is infinite dimensional. However, we believe that such a result can be obtained by looking at $\left.I\right|_{S_{l}}$. In preparation for a future work carrying out this idea, we prove the following theorem in Section 4

Theorem 1.6. If $(a, b) \in \mathrm{II}_{l} \backslash \Sigma$, then

$$
C_{q}(I, 0) \cong \begin{cases}H^{d_{l}-q-1}\left(\widehat{S}_{l}^{+}\right), & q \neq d_{l}-1, \\ H^{0}\left(\widehat{S}_{l}^{+}\right) / \mathbb{Z}, & q=d_{l}-1,\end{cases}
$$

where $\widehat{S}_{l}^{+}=\left\{u \in \widehat{S}_{l}(a, b): I(u)>0\right\}$.

Notation. We will use the customary notation

$$
I^{\alpha}:=\{u \in H: I(u) \leq \alpha\}, \quad \alpha \in \mathbb{R},
$$

for the sublevel sets.

\section{Proof of Theorem 1.3}

Let

$$
J(u, t)=I(u, \gamma(t)), \quad u \in H, t \in[0,2],
$$

where $\gamma$ is as in Definition 1.2 Then $J(\cdot, 0)=I_{p}, J(\cdot, 2)=I(\cdot, a, b)=I$, and the $C_{*}(J(\cdot, t), 0)$ are independent of $t \in(0,2]$, so

$$
C_{q}(I, 0) \cong C_{q}(\tilde{I}, 0)
$$

where $\tilde{I}=J(\cdot, 1)$. By (iii), $\gamma(t) \in \mathrm{I}_{l 1} \cup \mathrm{II}_{l}$ for $t>0$ sufficiently small, so both sides of (1.3) are zero for $q \geq d_{l}$ by Theorem 1.1 and hence we may assume that $q \leq d_{l}-1$.

Fix $\alpha<0$. Then

$$
C_{q}(\tilde{I}, 0) \cong H_{q}\left(H, \tilde{I}^{\alpha}\right)
$$

since 0 is the only critical point of $\tilde{I}$. By (iii), $J(u, t)$ is nondecreasing in $t$ on $[0,1]$, so $\tilde{I}^{\alpha} \subset I_{p}^{\alpha} \subset I_{p}^{0} \backslash K_{p}$. We claim that $\tilde{I}^{\alpha}$ is a strong deformation retract of $I_{p}^{0} \backslash K_{p}$. To see this let $\eta=\eta(u, t)$ be the flow generated by

$$
\left\{\begin{aligned}
\frac{d \eta}{d t} & =\frac{\alpha-I_{p}(u)-\partial_{t} J(\eta, t)}{\left\|\partial_{u} J(\eta, t)\right\|^{2}} \partial_{u} J(\eta, t) \quad \text { for } t \in[0,1], \\
\eta(u, 0) & =u \quad \text { on } I_{p}^{0} \backslash\left(K_{p} \cup \tilde{I}^{\alpha}\right) .
\end{aligned}\right.
$$

Then

$$
\frac{d}{d t} J(\eta, t)=\left(\partial_{u} J(\eta, t), \frac{d \eta}{d t}\right)+\partial_{t} J(\eta, t)=\alpha-I_{p}(u),
$$

$$
J(\eta(u, t), t)=(1-t) I_{p}(u)+\alpha t
$$


In particular, $I_{p}(\eta)=J(\eta, 0) \leq J(\eta, t)<0$ for $t \in(0,1]$ since $I_{p}(u) \leq 0$, so the flow stays in $I_{p}^{0} \backslash K_{p}$. Also, $\tilde{I}(\eta(u, 1))=J(\eta(u, 1), 1)=\alpha$. Moreover, at $t=0$,

$$
\begin{aligned}
& \alpha-I_{p}(u)-\partial_{t} J(\eta, t)=\alpha-I_{p}(u)-\partial_{t} J(u, 0)=\alpha-I\left(u, p+\gamma^{\prime}(0)\right) \\
& \quad=\alpha-\tilde{I}(u)
\end{aligned}
$$

by (iii), so $\eta(u, t)=u$ for all $t \in[0,1]$ if $\tilde{I}(u)=\alpha$. It follows that $\eta$ can be continuously extended to $\left(I_{p}^{0} \backslash K_{p}\right) \times[0,1]$ by setting $\eta(u, t)=u$ on $\tilde{I}^{\alpha} \times[0,1]$, defining a strong deformation retraction of $I_{p}^{0} \backslash K_{p}$ onto $\tilde{I}^{\alpha}$. Hence

$$
H_{q}\left(H, \tilde{I}^{\alpha}\right) \cong H_{q}\left(H, I_{p}^{0} \backslash K_{p}\right) .
$$

Since $p \in C_{l 2}$,

$$
I_{p}(u) \leq 0, \quad u \in S_{l 1},
$$

and

$$
K_{p}=\left\{u \in S_{l 1}: I_{p}(u)=0\right\} .
$$

Moreover, $I_{p}(v+w)$ is convex in $w \in M_{l}$ for fixed $v \in N_{l}$ and $I_{p}(u+w)>0$ for $u \in K_{p}, w \in M_{l} \backslash\{0\}$. Hence the mapping $\left(I_{p}^{0} \backslash K_{p}\right) \times[0,1] \rightarrow I_{p}^{0} \backslash K_{p}$,

$$
(v+w, t) \mapsto v+(1-t) w+t \tau(v),
$$

is a strong deformation retraction of the pair $\left(H, I_{p}^{0} \backslash K_{p}\right)$ onto the pair $\left(S_{l 1}, S_{l 1} \backslash K_{p}\right)$. Since $S_{l 1}$ is a radial manifold and $I$ is positive homogeneous, $\left(S_{l 1}, S_{l 1} \backslash K_{p}\right)$ is homotopic to $\left(B_{l 2}, \widehat{S}_{l 1} \backslash \widehat{K}_{p}\right)$ where $B_{l 2}=\left\{u \in S_{l 1}:\|u\| \leq 1\right\}$ and $\widehat{S}_{l 1}=\partial B_{l 2}$. Hence

$$
H_{q}\left(H, I_{p}^{0} \backslash K_{p}\right) \cong H_{q}\left(B_{l 2}, \widehat{S}_{l 1} \backslash \widehat{K}_{p}\right) .
$$

Combining (2.2), (2.3), (2.8), and (2.12) we have

$$
C_{q}(I, 0) \cong H_{q}\left(B_{l 2}, \widehat{S}_{l 1} \backslash \widehat{K}_{p}\right) .
$$

Now consider the exact sequence of the triple $\left(B_{l 2}, \widehat{S}_{l 1}, \widehat{S}_{l 1} \backslash \widehat{K}_{p}\right)$ :

$$
\begin{aligned}
& \cdots \longrightarrow H_{q+1}\left(B_{l 2}, \widehat{S}_{l 1} \backslash \widehat{K}_{p}\right) \longrightarrow H_{q+1}\left(B_{l 2}, \widehat{S}_{l 1}\right) \longrightarrow H_{q}\left(\widehat{S}_{l 1}, \widehat{S}_{l 1} \backslash \widehat{K}_{p}\right) \\
& \longrightarrow H_{q}\left(B_{l 2}, \widehat{S}_{l 1} \backslash \widehat{K}_{p}\right) \longrightarrow H_{q}\left(B_{l 2}, \widehat{S}_{l 1}\right) \longrightarrow \cdots .
\end{aligned}
$$

Since $B_{l 2}$ is homeomorphic to the unit ball in $N_{l}$,

$$
H_{q}\left(B_{l 2}, \widehat{S}_{l 1}\right)= \begin{cases}\mathbb{Z}, & q=d_{l}, \\ 0, & q \neq d_{l},\end{cases}
$$

so it follows that

$$
H_{q}\left(B_{l 2}, \widehat{S}_{l 1} \backslash \widehat{K}_{p}\right) \cong H_{q}\left(\widehat{S}_{l 1}, \widehat{S}_{l 1} \backslash \widehat{K}_{p}\right), \quad q<d_{l}-1 .
$$

Since $H_{d_{l}}\left(B_{l 2}, \widehat{S}_{l 1} \backslash \widehat{K}_{p}\right) \cong C_{d_{l}}(I, 0)=0$, for $q=d_{l}-1$ we get the short exact sequence

SO

$$
0 \longrightarrow \mathbb{Z} \longrightarrow H_{d_{l}-1}\left(\widehat{S}_{l 1}, \widehat{S}_{l 1} \backslash \widehat{K}_{p}\right) \longrightarrow H_{d_{l}-1}\left(B_{l 2}, \widehat{S}_{l 1} \backslash \widehat{K}_{p}\right) \longrightarrow 0
$$

$$
H_{d_{l}-1}\left(B_{l 2}, \widehat{S}_{l 1} \backslash \widehat{K}_{p}\right) \cong H_{d_{l}-1}\left(\widehat{S}_{l 1}, \widehat{S}_{l 1} \backslash \widehat{K}_{p}\right) / \mathbb{Z}
$$


Since

$$
H_{q}\left(\widehat{S}_{l 1}, \widehat{S}_{l 1} \backslash \widehat{K}_{p}\right) \cong H^{d_{l}-q-1}\left(\widehat{K}_{p}\right)
$$

by the Alexander duality theorem, (1.3) follows from (2.13), (2.16), and (2.18).

\section{Proof of Theorem 1.5}

Lemma 3.1. There are continuous and positive homogeneous functions $\xi: E\left(\lambda_{l}\right)$ $\rightarrow N_{l-1}, \eta: E\left(\lambda_{l}\right) \rightarrow M_{l}$ such that

$$
S_{l}=\left\{\xi(y)+y+\eta(y): y \in E\left(\lambda_{l}\right)\right\} .
$$

Proof. We have to show that for each $y \in E\left(\lambda_{l}\right)$, there is exactly one $u=v+y+w \in$ $N_{l-1} \oplus E\left(\lambda_{l}\right) \oplus M_{l}$ that is in $S_{l}$. Fix $y \in E\left(\lambda_{l}\right)$ and let

$$
L(v, w)=I_{p}(u)
$$

Then $u \in S_{l 1}$ if and only if

$$
L(v, w)=\inf _{h \in M_{l}} L(v, h),
$$

while $u \in S_{l 2}$ if and only if

$$
L(v, w)=\sup _{g \in N_{l-1}} L(g, w) .
$$

Thus $u \in S_{l}$ if and only if

$$
L(v, h) \geq \alpha, \quad h \in M_{l}, \quad L(g, w) \leq \alpha, \quad g \in N_{l-1},
$$

where

$$
\alpha=\sup _{g \in N_{l-1}} \inf _{h \in M_{l}} L(g, h)=\inf _{h \in M_{l}} \sup _{g \in N_{l-1}} L(g, h) .
$$

Let $u_{i}=v_{i}+y+w_{i} \in S_{l}, i=1,2$, so

$$
L\left(v_{i}, h\right) \geq \alpha, \quad h \in M_{l}, \quad L\left(g, w_{i}\right) \leq \alpha, \quad g \in N_{l-1} .
$$

In particular

$$
L\left(v_{i}, w_{1}\right) \geq \alpha, \quad i=1,2 .
$$

Since $L(v, w)$ is strictly concave in $v$, if $v_{1} \neq v_{2}$ this implies

$$
L\left((1-t) v_{1}+t v_{2}, w_{1}\right)>\alpha, \quad t \in(0,1),
$$

contradicting (3.7). Similarly $w_{1}=w_{2}$.

We are now ready to prove Theorem 1.5. Since $\Gamma_{p} \neq \emptyset$ and $p \notin C_{l 1}$, using (iii) of Definition 1.2 we can take a point $(a, b) \in \Gamma_{p} \cap \mathrm{II}_{l}$. Then

$$
C_{d_{l-1}}(I, 0)=0
$$

by Theorem 1.1, so

$$
i_{d_{l-1}}(p)=0
$$


by Theorem 1.3 Since $\widehat{K}_{p} \subset \widehat{S}_{l}$ and $\widehat{S}_{l}$ is homeomorphic to the $\left(d_{l}-d_{l-1}-1\right)$ dimensional sphere by Lemma 3.1, this implies that $\widehat{K}_{p}$ is a proper subset of $\widehat{S}_{l}$. If $\lambda_{l}$ is simple, $\mathrm{II}_{l} \cap \Sigma=\emptyset$ by Gallouët and Kavian [3], so $\Gamma_{p} \neq \emptyset$ for any $p \in Q_{l} \cap C_{l 2}$.

\section{Proof of Theorem 1.6}

Since 0 is the only critical point of $I$,

$$
C_{q}(I, 0) \cong H_{q}\left(H, I^{0} \backslash\{0\}\right) .
$$

Arguments similar to those in the proof of Theorem 1.3 show that (2.11) defines a strong deformation retraction of $\left(H, I^{0} \backslash\{0\}\right)$ onto $\left(S_{l 1},\left(I^{0} \cap S_{l 1}\right) \backslash\{0\}\right)$ and

$$
H_{q}\left(S_{l 1},\left(I^{0} \cap S_{l 1}\right) \backslash\{0\}\right) \cong \begin{cases}H^{d_{l}-q-1}\left(\widehat{S}_{l 1}^{+}\right), & q \neq d_{l}-1, \\ H^{0}\left(\widehat{S}_{l 1}^{+}\right) / \mathbb{Z}, & q=d_{l}-1,\end{cases}
$$

where $\widehat{S}_{l 1}^{+}=\left\{u \in \widehat{S}_{l 1}: I(u)>0\right\}$. For $u \in N_{l}$, let $\zeta(u)=u+\tau(u)$. Then $\widehat{S}_{l 1}=\left\{\zeta(v+y): v+y \in N_{l-1} \oplus E\left(\lambda_{l}\right),\|\zeta(v+y)\|=1\right\}$. We claim that

$$
(\zeta(v+y), t) \mapsto \frac{\zeta((1-t) v+t \xi(y)+y)}{\|\zeta((1-t) v+t \xi(y)+y)\|}, \quad \zeta(v+y) \in \widehat{S}_{l 1}^{+}, t \in[0,1],
$$

where $\xi$ is as in Lemma 3.1, defines a strong deformation retraction of $\widehat{S}_{l 1}^{+}$onto $\widehat{S}_{l}^{+}$. We have to show that

$$
I(\zeta((1-t) v+t \xi(y)+y))>0 .
$$

First note that

$$
\begin{aligned}
I(\zeta(\xi(y)+y)) & \geq I(v+y+\tau(\xi(y)+y)) \\
& \geq I(\zeta(v+y)) .
\end{aligned}
$$

So, since $I(v+y+w)$ is concave in $v$ for any $w \in M_{l}$,

$$
\begin{aligned}
I((1-t) v+t \xi(y)+y+w) & \geq(1-t) I(v+y+w)+t I(\xi(y)+y+w) \\
& \geq(1-t) I(\zeta(v+y))+t I(\zeta(\xi(y)+y)) \\
& \geq I(\zeta(v+y))>0,
\end{aligned}
$$

and (4.4) follows. This completes the proof of Theorem 1.6.

\section{REFERENCES}

[1] E. N. Dancer. Multiple solutions of asymptotically homogeneous problems. Ann. Mat. Pura Appl. (4), 152:63-78, 1988. MR 90i:35095

[2] E. N. Dancer. Remarks on jumping nonlinearities. In Topics in nonlinear analysis, pages 101-116. Birkhäuser, Basel, 1999. CMP 2000:05

[3] T. Gallouët and O. Kavian. Résultats d'existence et de non-existence pour certains problèmes demi-linéaires à l'infini. Ann. Fac. Sci. Toulouse Math. (5), 3(3-4):201-246 (1982), 1981. MR 83m:35058

[4] K. Perera and M. Schechter. A generalization of the Amann-Zehnder theorem to nonresonance problems with jumping nonlinearities. to appear in NoDEA Nonlinear Differential Equations Appl. 7 (2000) no. 3.

[5] K. Perera and M. Schechter. Type II regions between curves of the Fučík spectrum and critical groups. Topol. Methods Nonlinear Anal., 12(2):227-243, 1998. MR 2000h:35052 
[6] M. Schechter. Resonance problems with respect to the Fučík spectrum. Trans. Amer. Math. Soc., 352:4195-4205, 2000. CMP 2000:14

[7] M. Schechter. The Fučík spectrum. Indiana Univ. Math. J., 43(4):1139-1157, 1994. MR 96c:35063

[8] M. Schechter. Type (II) regions between curves of the Fučík spectrum. NoDEA Nonlinear Differential Equations Appl., 4(4):459-476, 1997. MR 99b:35071

Department of Mathematical Sciences, Florida Institute of Technology, Melbourne, FLORIDA 32901-6975

E-mail address: kperera@winnie.fit.edu

URL: http://winnie.fit.edu/ ${ }^{\sim}$ kperera/

Department of Mathematics, University of California-Irvine, Irvine, California 92697-3875

E-mail address: mschecht@math.uci.edu

$U R L:$ http://www .math.uci.edu/mschecht.html 